\title{
Primary temporal bone angiosarcoma: a case report
}

\author{
Martin Scholsem ${ }^{1}$, Daniel Raket ${ }^{2}$, Pierre Flandroy ${ }^{3}$, Raf Sciot ${ }^{4}$ and Manuel Deprez ${ }^{5}$ \\ ${ }^{1}$ Department ofNeurosurgery, University Hospital of Liège, Liège; ${ }^{2}$ Department of Neurosurgery, Cliniques Saint Joseph, Liège; \\ ${ }^{3}$ Department of Radiology, University Hospital of Liège, Cliniques Saint Joseph Liège; ${ }^{4}$ Department of Pathology, University of Leuven, \\ Flanders; ${ }^{5}$ Laboratory of Neuropathology, University Hospital of Liège, Liège, Belgium
}

\section{Summary}

We present a rare case of temporal bone angiosarcoma diagnosed in a 26-year-old female patient at 36 week of pregnancy. The patient was referred with a 2 months history of left otalgia and tinnitus with a tender swelling above the mastoid. Cranial imaging studies showed a $7 \times 5 \times 4 \mathrm{~cm}$ hypervascularized mass located in the left middle fossa with lysis of the temporal bone and extension to the subcutis. After the baby was delivered by caesarean section, the patient entered the oncology protocol. Selective embolization of the feeding vessels was followed by gross total surgical resection using a combined supra- and infra-tentorial approach. Pathological findings were those of a poorly differentiated, highly malignant sarcoma with a large epitheloid component and immunohistochemical evidence of endothelial differentiation (CD31, Factor VIII related antigen, CD34), consistent with an angiosarcoma with epitheloid features. No extra-cranial tumor was found after extensive staging. The patient received adjuvant radiotherapy followed by a course of chemotherapy consisting of 6 cycles of paclitaxel. At 15 months follow-up, she developed multiple distant metastasis to a left postauricular lymph node and to the lungs and ribs. The patient was given a second line chemotherapy using doxorubicine and ifosfamide. Despite an initial good response, she died with metastatic disease 26 months after diagnosis. We present a rare case of primary temporal bone angiosarcoma and report our experience with a multimode therapeutic approach combining surgery, radiotherapy and chemotherapy.

Keywords : angiosarcoma ; chemotherapy ; cranial tumor ; pregnancy ; temporal bone

\section{Introduction}

Angiosarcomas are rare tumors accounting for less than $1 \%$ of all sarcomas, with a reported annual incidence of approximately $2-3$ cases per $1,000,000$ population [1,2].

Half of these sarcomas occur in the head and neck, mainly in the skin and subcutis and less than $2 \%$ are intracranial [3]. In this latter localization, they arise from the mesenchymal elements of the brain and its coverings but also from cranial bones [4-8]. They present either as the sole or minor component of a primary tumor or as a metastasis of a distant extracranial angiosarcoma [3,7-10].

Primary skull angiosarcomas are extremely rare and only 16 acceptable cases have been reported in the literature [11].

In this communication, we report the case of a patient presenting with tinnitus and left otalgia caused by a temporal bone angiosarcoma arising in the mastoid and extending in the subcutaneous tissue and in the middle and posterior cranial fossa.

\section{Case report}

History and examination

A 26-year-old Caucasian female was admitted to our institution with a 2-month history of tinnitus and left otalgia associated with a retroauricular swelling. The patient was at 36 week of an uncomplicated pregnancy and had no personal medical or surgical history. Her family history was unremarkable. 
Examination of the left mastoid region revealed a tender subcutaneous $2 \mathrm{~cm}$ mass with no abnormality of the overlying skin. Otoscopy showed a deformation of the tympanic membrane and of the external ear conduct by a voluminous angiomatous formation.

There was no neurological deficit and the hearing testing was normal. There was no enlargement of local and regional lymph nodes. A detailed examination of the whole body failed to detect any other cutaneous or subcutaneous lesion.

Brain CT scan showed a $7 \times 5 \times 4 \mathrm{~cm}$ hyperdense mass located mainly in the left middle fossa with extension to the posterior fossa through the tentorium cerebelli (Figure 1).

Figure 1. Computed tomographic (CT) scan after intravenous administration of contrast showing a left hyperdense tumor with both supratentorial and infratentorial extension.

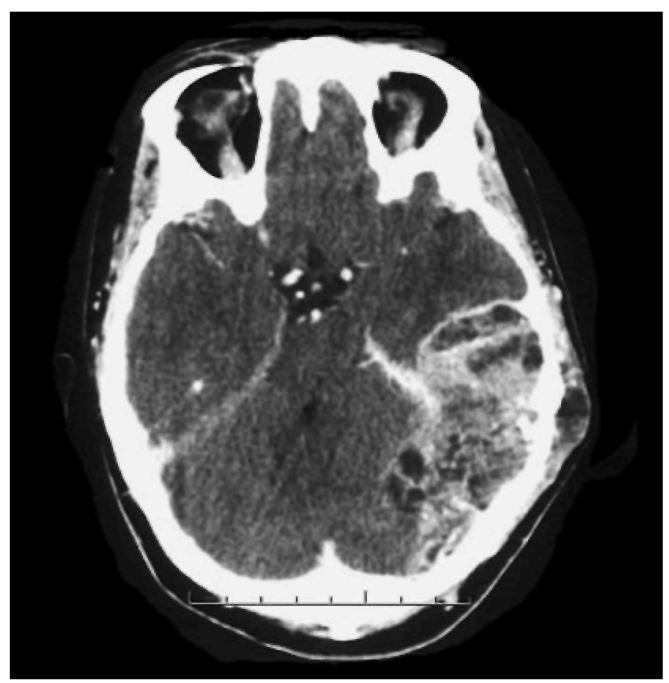

High resolution temporal bone CT scan showed a lysis of the mastoid by a tumor with soft tissue density extending to the squamous part of the temporal bone and the subcutis. The lesion contained several cystic areas and was markedly enhanced after contrast injection.

Brain MRI was consistent with the presence of a well-demarcated expansive lytic lesion involving the petrous and squamous portion of the temporal bone and extending both to intracranial and extracranial compartment.

The tumor was hyperintense on T1-weigthed images with several low signal areas (Figure 2). The echo gradient sequences suggested the deposition of haemosiderin in the tumor.

Figure 2. Axial T1-weighted MR images: the left temporal tumor is hyperintense with scattered isointense regions (left) and shows marked diffuse enhancement after contrast injection (middle). Coronal T2-weighted MR image, showing the supra- and infra-tentorial components of the tumor and its relation to the tentorium (right).
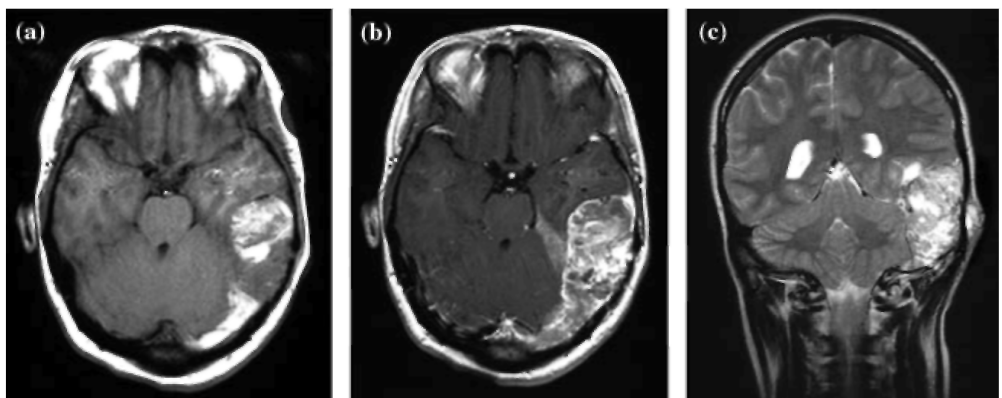
It was decided to deliver the baby by caesarean section before proceeding to invasive studies. The newborn was a healthy $3.5 \mathrm{~kg}$ boy.

Arteriography of the brain vessels showed a hyper-vascularized tumor compressing the left temporal lobe and lifting up the temporal branches of the middle cerebral artery.

Angiography of the left common carotid showed that the blood supply was mainly derived from the middle meningeal artery and from some branches of the posterior auricular artery and the occipital artery. Left internal carotid angiograms showed an additional feeding by the tentorium cerebelli artery coming from the carotid siphon (Figure 3). The left lateral sinus was thrombosed.

Figure 3. Lateral view of left common carotid angiogram: the lesion is primarily fed by the middle meningeal artery and by branches from the posterior auricular artery and the occipital artery, with additional supply from the tentorium cerebelli artery(left). The late arterial phase demonstrates the tumor hypervascularization (right).
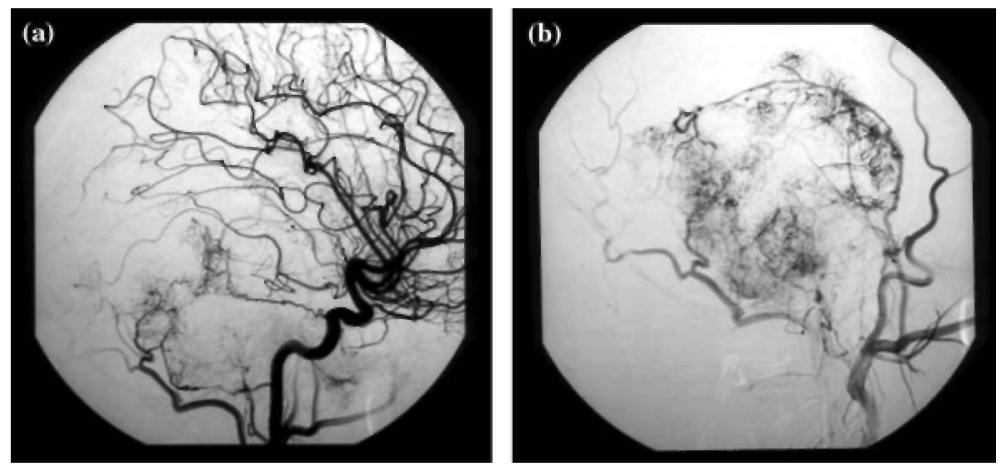

Surgery

Embolization of the feeding vessels was realized $24 \mathrm{~h}$ before surgery. The tumor was exposed by a left combined suboccipital transtemporal approach. An L-shaped incision was used, starting in the temporal region slightly above the ear, curving posteriorly, and finishing parallel and slightly below the mastoid process. The temporal and suboccipital musculature was incised, so as to expose the temporobasal and lateral suboccipital skull. A bloody and serous liquid was seen pouring through the squamous part of the remodelled temporal bone. We performed a combined supra/infratentorial craniectomy and resected under microscopic control a mass which was destructing the petrous bone and infiltrating the dura and the tentorium cerebelli. The tumor appeared grayish, friable and was fed by many congestive vessels. A gross total resection of the mass was performed and the dural defect was repaired with artificial dura. The cranial vault defect was finally reshaped with methylmethacrylate.

\section{Pathological findings}

Peroperative frozen sections showed a largely necrotic spindle cell tumor with profuse mitotic activity consistent with a high grade sarcoma.

Permanent sections from formalin-fixed paraffin-embedded tissue were stained with H\&E, PAS, reticulin stain (Foot's method) and antibodies specific for Vimentin (1:100, Dako, Glostrup, Denmark), Actin (1:100, Dako), Desmin (1:80, Dako), Epithelial Membrane Antigen (EMA) (1:20, Dako), Keratin (KL-1) (1:50, Immunotech, Marseille, France), S-100 protein (1:2000, Dako), CD68 (1:200, Dako), Glial Fibrillary Acidic Protein (GFAP) (1:500, Dako), CD31 (1:80, Dako), CD34 (1:1, RTU, NeoMarkers,Westinghouse Dr. Fremont, CA, USA), Ulex Europeaus (1:200 + 1:400, Dako), Factor VIII (1:1, RTU, Dako) and Ki-67 (1:8, RTU, Dako).

The tumor consisted of solid nests or large sheets of spindle or epitheloid cells interspersed between and around pre-existing vessels with flat endothelium (Figure 4a, c and d). The tumor cells were large, pleomorphic and showed a moderately abundant eosinophilic cytoplasm and a round to oval nucleus with one or two prominent 
nucleoli. Mitotic figures were numerous and frequently abnormal. Mitotic counts frequently exceeded 4-5 per high power field. Necrosis and apoptosis were readily found throughout the tumor.

Reticulin fibers were abundant delineating trabeculae or isolated tumor cells (Figure 4b).

Immunohistochemistry showed a strong cytoplasmic staining of tumor cells for Vimentin, CD31 and Factor VIII related antigen (Figure 4e and f). The immunostaining for CD34 and Ulex was weaker and focal. There was also a faint diffuse cytoplasmic and granular staining for EMA. Cells were negative for S-100, GFAP, Actin, Desmin, and KL-1. The proliferation index was extremely high with $80-90 \%$ of nuclei positive for Ki-67.

A final diagnosis of angiosarcoma with epitheloid features was proposed and the search for an possible extracranial primary tumor was recommended.

Figure 4. (a, c, d) H\&E stains of the tumor specimen (original magnification (a) $200 \times,(c, d) 400 \times$ ): large sheets of spindle or epitheloid cells are interspersed between pre-existing vessels with flat endothelium. The mitotic activity is high and there are numerous areas of necrosis (arrows) (b) there is abundant reticulin deposition between tumor cells (Foot's method, original magnification 200×). (e, f) Immunohistochemistry (original magnification $200 \times$ ) shows strong membranous positive staining for CD31 (e) and cytoplasmic positivity for Factor VIII (f).
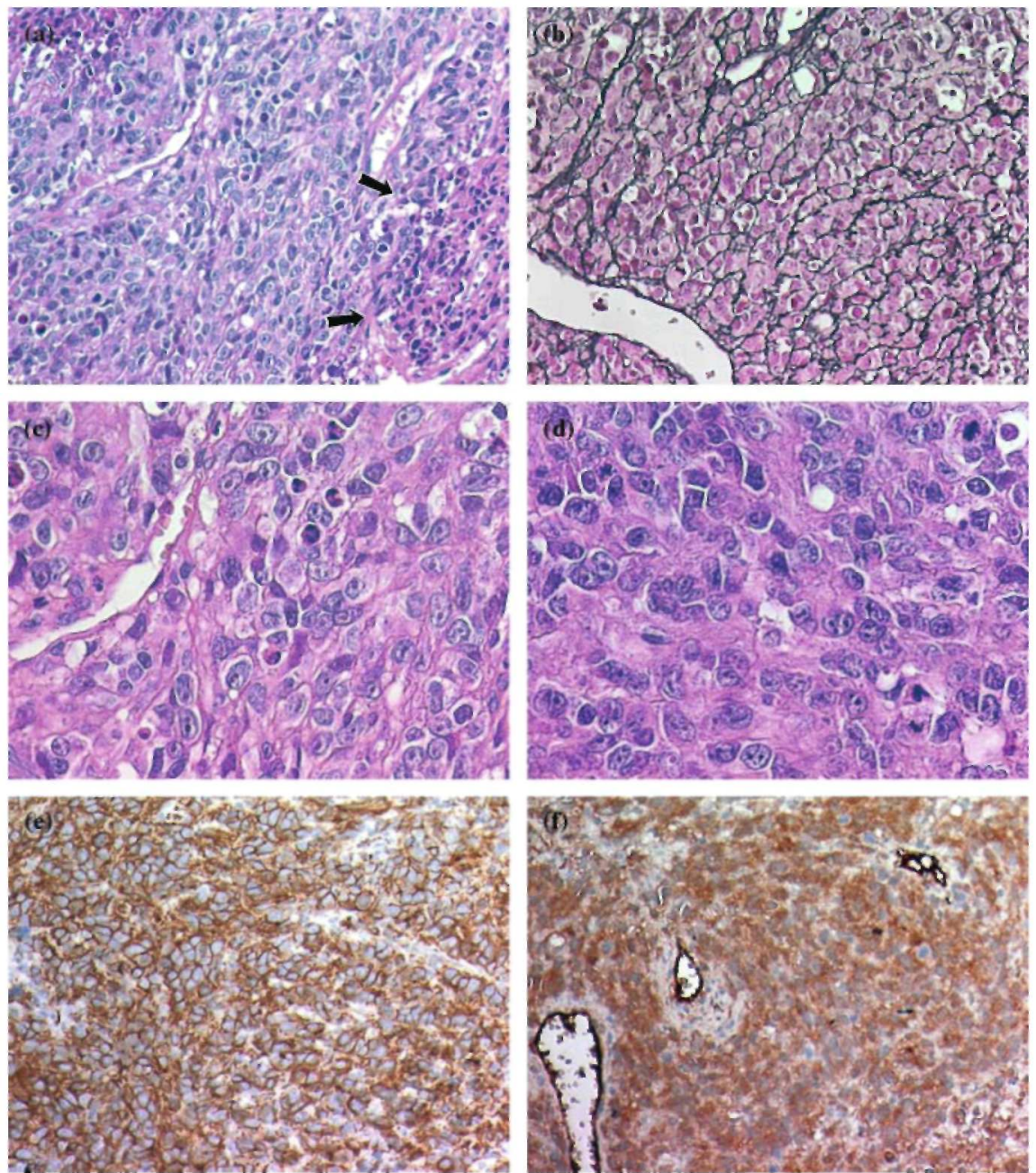


\section{Postoperative course}

The patient had an uneventful postoperative course and did not exhibit any neurological deficit. Postoperative brain MRI confirmed that the tumor had been totally resected. The systemic exploration included a thoracoabdominal CT scan, bone scintigraphy and senologic examination. No other tumor was found. The patient was included in a protocol of whole brain radiotherapy consisting of a total of 60 Gy delivered in 30 daily fractions of 2 Gy each. She subsequently received an adjuvant chemotherapy consisting of 6 cycles of paclitaxel at the dose of $175 \mathrm{mg} / \mathrm{m}^{2}$.

The patient remained well during 15 months with no sign of recurrence and the brain MRI did not show any regrowth or pathological enhancement.

The patient was still asymptomatic at her latest examination. However, the discovery of a left postauricular adenopathy prompted a search for distant metastasis. Total body ${ }^{18}$ FDG-PET followed by thoracic CT scan and chest X-ray revealed the presence of multiple metastasis to the lung and ribs.

The patient was given a second line chemotherapy using doxorubicine at the dose of $30 \mathrm{mg} / \mathrm{m}^{2}$ and ifosfamide at the dose of $3750 \mathrm{mg} / \mathrm{m}^{2}$. She initially showed a good response with marked reduction of the adenopathy and other metastasis. However, she died of metastatic disease 26 months after the diagnosis.

\section{Discussion}

Angiosarcoma is a rare malignant soft tissue tumor with endothelial differentiation that can involve soft tissue or bone. They account for only $0.5-1 \%$ of malignant primary bone tumors [12-15]. The majority of bone angiosarcomas occur in the limbs and much less frequently in the ribs, pelvis and vertebra. The skull is an extremely unusual site of occurrence with only 16 cases reported in the literature [11]. Frontal and parietal bones are more commonly involved than the occipital, sphenoid and temporal bones $[12,15,16]$. Reviewing previous literature, we found only seven cases of temporal bone angiosarcoma [11,13,14,17-20].

Unlike extracranial angiosarcomas in which radiation therapy, arsenic exposure and previous trauma have been implicated as predisposing factors, no risk factors were found for primary skull angiosarcomas [11,16]. Skull angiosarcomas are often unicentric tumors although a case of multiple angiosarcoma has been also described [16].

In previous reports of skull angiosarcoma, most cases occurred in the third decade with a marked male predominance $[2,12,16]$.

Although skull angiosarcoma often present as an indolent mass or swelling, in the temporal bone, the common clinical manifestations are tinnitus, hearing impairment and otalgia with a swelling in the temporal region $[12,13,17-19]$, as in our patient.

The radiographic findings of skull angiosarcoma usually demonstrate a well-demarcated lytic, hypervascularized and hemorrhagic mass [14]. These neuroradiological features are not pathognomonic and may also be seen in other sarcomas or metastasis [6,9].

On radiological basis, we believe that the current angiosarcoma originated in the skull because of the destruction of the temporal bone and the main intracranial position. An origin in the subcutis is highly improbable considering the absence of skin change, the age of the patient and the presentation mode.

However, rare cases of meningeal tumor have also been described and a derivation from the mesenchymal cells of the meninges cannot be rule out [8].

Angiosarcomas exhibit variable malignant behavior related to their microscopic appearance and degree of vascular differentiation. In poorly differentiated tumors, immunohistochemistry is mandatory for the identification of an endothelial lineage [6]. Antibodies currently used are Factor VIII-related antigen and CD 31, which are specific markers for endothelial cells [6,9, 11-14,17,21].

The clinical course is always characterized by the rapid onset of symptoms and the high frequency of local recurrence or metastasis [6]. Patients with skull angiosarcoma have a poor outcome although long-term survivors have been occasionally seen [22]. Their prognosis is worse prognosis than for other bone angiosarcomas because 
of the frequent secondarily involvement of the meninges and the brain and the difficulty to perform a complete surgical resection.

Angiosarcomas have a strong metastatic potential and an extensive staging must be conducted in all cases at the time of diagnosis and, as suggested by Bourekas et al. [12], the follow-up should include regular bone CT scan, chest radiography and cerebral imaging. Because of the rarity of cranial bone angiosarcomas, optimal management has not been defined. The most effective treatment is the complete surgical removal of the tumor but adequate surgical margins are often very difficult to secure. Since angiosarcomas are hypervascularized, preoperative embolization facilitates near total excision of the tumor with minimal blood loss. As previously mentioned, our patient received adjuvant radiotherapy followed by chemotherapy. The role of adjuvant treatment is not well defmed. Radiotherapy has been given as an adjunct to surgical therapy or as palliative treatment in numerous cases of skull angiosarcoma $[13,16,18,19]$ including three cases of temporal angiosarcoma $[13,18,19]$ but its effectiveness has not been proven yet. In the case we report, radiotherapy was given after surgery because of the important infiltration of the petrous bone by the tumor. It is of interest that in our case, no sign of local recurrence has been detected 26 months after surgery suggesting that radiotherapy may enhance local control.

There are very few data in the literature regarding the benefit of chemotherapy. Recent data suggest that adjuvant chemotherapy with paclitaxel improves the condition of patients with soft-tissue sarcoma of the face or scalp $[23,24]$. In the case of skull angiosarcoma, however, the effectiveness of chemotherapy has not been substantiated. The use of paclitaxel was well tolerated in our patient but she developed bone and pulmonary metastasis and died 20 months after completion of the chemotherapy.

In conclusion, we present a rare case of primary temporal bone angiosarcoma and report our experience with a multimode therapeutic approach combining surgery, radiotherapy and chemotherapy.

\section{References}

1. Enzinger FM, Weiss SW (eds) Soft Tissue Tumors. 3rd edn. Mosby, St. Louis, MO 1995, pp. 648-677.

2. Fedok FG, Levin RJ, Maloney ME, Tipirneni K: Angiosarcoma: current review .Am J Otolaryngol 20(4): 223-231, 1999

3. Naka N, Ohsawa M, Tomita Y, Kanno H, Uchida A, Aozasa K: Angiosarcoma in Japan: a Review of 99 Cases. Cancer 75(4): 989-996, 1995

4. Cookston M, Cotter GW, Schlitt M, Bastian FO: Primary angiosarcoma of the brain. South Med J. 84(4): 517-520, 1991

5. Kirk IR, Dominguez R, Castillo M: Congenital primary cerebral angiosarcoma: CT, US, and MR findings. Pediatr Radiol. 22(2): 134135,1992

6. Mena H, Ribas J, Enzinger F, Parisi J: Primary angiosarcoma of the central nervous system: study of eight cases and review of the literature. J Neurosurg 75: 73-76, 1991

7. Paulus W, Slowik F, Jellinger K: Primary intracranial sarcomas: histopathological features of 19 cases. Histopathology 18(5): 395-402, 1991

8. Russell DS, Rubinstein LJ: Pathology of Tumours of the Nervous System, 5th edn. Williams \& Wilkins, Baltimore, 1989, pp. 657-658.

9. Antoniadis C, Selviaridis P, Zaramboukas T, Fountzilas G: Primary angiosarcoma of the brain: case report. Neurosurgery 38: 583-586, 1996

10. Flemming JFR, Deck JHN, Bernstein M: Intracranial sarcomas. In: Wilkins RH, Rengachary SS (eds) Neurosurgery. McGraw-Hill, New York, 1985, pp. 1030-1036

11. Lopes M, Duffau H, Fleuridas G: Primary spheno-orbital angiosarcoma: case report and review of the literature. Neurosurgery 44(2): 405-407; Discussion 407-408, 1999

12. Bourekas E, Cohen M, Kamen C, Tarr R, Lanzieri C, Lewin J: Maligant hemangioendothelioma (angioasarcoma) of the skull: plain film, CT and MR Appearance. AJNR Am J Neuroradiol 17: 1946-1948, 1996

13. Goldstein WS, Bowen BC, Balkany T: Malignant hemangioendothelioma of the temporal bone masquerading as glomus tympanicum. Ann Otol Rhinol Laryngol 103(2): 156-159, 1994

14. Ibarra RA, Kesava P, Hallet KK, Bogaev C: Hemangioendothelioma of the temporal bone with radiologic findings resembling hemangioma. AJNR Am J Neuroradiol 22(4): 755-758, 2001 
Published in : Journal of neuro-oncology (2005), vol. 75, pp. 121-125

Status : Postprint (Author's version)

15. Volpe R, Mazabraud A: Hemangioendothelioma (angiosarcoma) of bone: a distinct pathologic entity with an unpredictable course? Cancer 15; 49(4): 727-736, 1982

16. Chou YC, Chang YL, Harnod T, Chen WF, Su CF, Lin SZ, Hsu YH, Yen PS, Lee CC: Primary angiosarcoma of the cranial vault: a case report and review of the literature. Surg Neurol 61(6): 575-579, 2004

17. Eliashar R, Saah D, Osin P, Sichel JY: Hemangioendothelioma of the temporal bone in a child. Int J Pediatr Otorhinolaryngol 40(1): $67-71,1997$

18. Joachims HZ, Cohen Y: Hemangioendothelioma of the mastoid bone. Laryngoscope 84(3): 454-458, 1974

19. Kinkade JM: Angiosarcoma of the petrous portion of the temporal bone. Report of a case. Ann Otol Rhinol Laryngol 57: 235-240, 1948

20. Masieh M: Haemangiosarcoma of the petrous temporal bone. J Laryngol Otol 94(2): 205-210, 1980

21. Muhlau M, Schlegel J, Von Einsiedel HG, Conrad B, Sander D: Multiple progressive intracerebral hemorrhages due to an angiosarcoma: a case report. Eur J Neurol 10(6): 741-742, 2003

22. Unni KK, Ivins JC, Beabout JW, Dahlin DC: Hemangioma, hemangiopericytoma, and hemangioendothelioma (angiosarcoma) of bone. Cancer 27(6): 1403-1414, 1971

23. Casper ES, Waltzman RJ, Schwartz GK, Sugarman A, Pfister D, Ilson D, Woodruff J, Leung D, Bertino JR: Phase II trial of paclitaxel in patients with soft-tissue sarcoma.Cancer Invest 16(7): 442-446, 1998

24. Fata F, O'Reilly E, Ilson D, Pfister D, Leffel D, Kelsen DP, Schwartz GK, Casper ES: Paclitaxel in the treatment of patients with angiosarcoma of the scalp or face. Cancer 86(10): 2034-2037, 1999 